\title{
IMPACTE DA PANDEMIA POR COVID-19 NOS ENFERMEIROS DE REABILITAÇ̃̃O PORTUGUESES
}

\section{IMPACTO DE LA PANDEMIA POR COVID-19 EN LOS ENFERMEROS DE REHABILITACIÓN PORTUGUESES}

\author{
IMPACT OF PANDEMIC BY COVID-19 ON PORTUGUESE REHABILITATION NURSES
}

DOI 10.33194/rper.2020.v3.s2.15.5842 | Submetido 22/11/2020 | Aprovado 15/12/2020

\author{
José Miguel Dos Santos Castro Padilha ${ }^{1,2} \mathbb{1}$; Rui Pedro Marques da Silva ${ }^{3}[0$ \\ 1 - Escola Superior de Enfermagem do Porto; 2 - PI-Tech4EduSim/ CINTESIS; 3 - ACeS Maia/Valongo
}

\begin{abstract}
RESUMO
Introdução: A pandemia por COVID-19 tem causado um impacte mundial significativo ao nível da saúde e ao nível socioeconómico. Paralelamente, tem causado uma sobrecarga nos sistemas de saúde e nos seus profissionais, entre os quais os enfermeiros de reabilitação. A real expressão desse impacte ao nível dos enfermeiros de reabilitação é desconhecida.
\end{abstract}

Objetivo: Avaliar o impacte da pandemia por COVID19 nos enfermeiros de reabilitação portugueses.

Método: Estudo observacional, descritivo e transversal, com uma amostra não probabilística de enfermeiros de reabilitação portugueses com desempenho de funções em qualquer tipologia de serviço nos três meses anteriores à recolha de dados. A recolha de dados realizou-se no início do terceiro trimestre de 2020 através de um questionário online disponibilizado por email.

Resultados: Amostra constituída por 146 enfermeiros especialistas em Enfermagem de reabilitação (EEER), dos quais $31 \%(n=45)$ teve de cessar a prestação de cuidados especializados durante a pandemia (aumento de 2,7x). Relativamente à satisfação com a qualidade dos cuidados prestados, a média antes do início da pandemia situava-se em 3,95 (SD $\pm 0,75)$ e durante a pandemia desceu para 2,9 (SD $\pm 1,11)$ (escala de Likert de 5 pontos). Dos participantes. $73,3 \%(n=107)$ referem que tiveram de seguir, em algum momento, orientações institucionais em desacordo com os seus princípios éticos e deontológicos, havendo 69,9\% $(n=102)$ que refere ter tido necessidade, em pelo menos uma situação, de priorizar a que pessoas doentes prestar cuidados. Durante a pandemia os EEER recorreram a colegas peritos e a recursos online para aumentar conhecimentos e capacidades em relação aos cuidados inerentes à pandemia.

Conclusão: Durante o primeiro pico pandémico por COVID 19 uma parte significativa dos EEER teve de assegurar apenas cuidados gerais. Os principais desafios enfrentados pelos EEER foram no domínio da organização e gestão dos cuidados devido à maior complexidade dos doentes, à maior carga burocrática, às mudanças no relacionamento com os colegas de trabalhos e à necessidade de balanço entre a vida profissional e pessoal. Verificou-se diminuição da satisfação com a qualidade dos cuidados prestados, bem como uma elevada percentagem de EEER que vivenciou desafios éticos e deontológicos. De forma a assegurar a sua formação contínua e para atualizar as competências e garantir a qualidade e segurança dos cuidados de enfermagem, os EEER revelaram dinamismo e apetência para a utilização das tecnologias da informação e comunicação.

Palavras-chave: enfermagem de reabilitação; enfermeiros de reabilitação; COVID-19; pandemia; impacte

\section{RESUMEN}

Introducción: La pandemia de COVID-19 ha tenido un impacto global significativo en la salud y el estado socioeconómico. Al mismo tiempo, ha provocado una sobrecarga en los sistemas de salud y sus profesionales, incluidas las enfermeras de rehabilitación. Se desconoce la expresión real de este impacto a nivel de enfermeras de rehabilitación.

Objetivo: evaluar el impacto de la pandemia COVID19 en las enfermeras de rehabilitación portuguesas.

Método: Estudio observacional, descriptivo y transversal, con una muestra no probabilística de enfermeras de rehabilitación portuguesas que desempeñaron funciones en cualquier tipo de servicio en los tres meses previos a la recolección de datos. La recopilación de datos se llevó a cabo a principios del tercer trimestre de 2020 a través de un cuestionario en línea enviado por correo electrónico.

Resultados: Muestra compuesta por 146 enfermeros especializados en Enfermería de Rehabilitación (EEER), de los cuales el 31\% $(n=45)$ tuvo que dejar de brindar atención especializada durante la pandemia (aumento de 2.7x). En cuanto a la satisfacción con la calidad de la atención brindada, el promedio antes del inicio de la pandemia fue de $3,95(D E \pm 0,75)$ y durante la pandemia bajó a $2,9(D E \pm 1,11)$ (escala de Likert de 5 puntos). De los participantes. El $73,3 \%(n=107)$ refiere que en algún momento tuvo que seguir pautas institucionales en desacuerdo con sus principios éticos y deontológicos, siendo el $69,9 \%(n=102)$ quien reportó haber tenido una necesidad, al menos una situación, 
para priorizar a qué personas enfermas cuidar. Durante la pandemia, EEER utilizó colegas expertos y recursos en línea para aumentar el conocimiento y las habilidades en relación con la atención inherente a la pandemia.

Conclusión: Durante el primer pico pandémico de COVID 19, una parte significativa de los EEER tuvo que garantizar solo atención general. Los principales retos a los que se enfrentó EEER fueron en el ámbito de la organización y gestión de la atención debido a la mayor complejidad de los pacientes, la mayor carga burocrática, los cambios en la relación con los compañeros de trabajo y la necesidad de conciliar la vida profesional y personal. Hubo una disminución en la satisfacción con la calidad de la atención brindada, así como un alto porcentaje de EEER que experimentaron desafíos éticos y deontológicos. Con el fin de asegurar su formación continua y actualizar sus competencias y garantizar la calidad y seguridad de los cuidados de enfermería, los EEER han mostrado dinamismo y disposición al uso de las tecnologías de la información y la comunicación.

Palabras clave: enfermería rehabilitadora; enfermeras de rehabilitación; COVID-19; pandemia; impacto

\section{ABSTRACT}

Introduction: The COVID-19 pandemic has had a significant global impact on health and socioeconomic status. At the same time, it has caused an overload on health systems and their professionals, including rehabilitation nurses. The real expression of this impact at the level of rehabilitation nurses is unknown.

Objective: To assess the impact of the COVID19 pandemic on Portuguese rehabilitation nurses.

Method: Observational, descriptive and cross-sectional study, with a non-probabilistic sample of Portuguese rehabilitation nurses who performed functions in any type of service in the three months prior to data collection. Data collection took place at the beginning of the third quarter of 2020 through an online questionnaire provided by email.

Results: Sample consisting of 146 nurses specialized in Rehabilitation Nursing (EEER), of which $31 \%$ ( $n=45$ ) had to cease providing specialized care during the pandemic (increase of $2.7 x$ ). Regarding satisfaction with the quality of care provided, the average before the start of the pandemic was 3.95 (SD \pm 0.75$)$ and during the pandemic it dropped to 2.9 ( $S D \pm 1.11$ ) (scale of 5-point Likert). Of the participants. 73.3\% $(n=107)$ refer that they had, at some point, to follow institutional guidelines in disagreement with their ethical and deontological principles, with $69.9 \%(\mathrm{n}=102)$ reporting having had a need, at least a situation, to prioritize which sick people to care for. During the pandemic, the EEER relied on expert colleagues and online resources to increase knowledge and skills regarding the care inherent in the pandemic.

Conclusion: During the first pandemic peak by COVID 19, a significant part of the EEERs had to ensure only general care. The main challenges faced by EEER were in the field of organization and management of care due to the greater complexity of patients, the greater bureaucratic burden, changes in the relationship with co-workers and the need to balance professional and personal life. There was a decrease in satisfaction with the quality of care provided, as well as a high percentage of EEER who experienced ethical and deontological challenges. In order to ensure their continuous training and to update their skills and guarantee the quality and safety of nursing care, the EEERs have shown dynamism and a willingness to use information and communication technologies.

Keywords: rehabilitation nursing; rehabilitation nurses; COVID-19; pandemic; impact

\section{INTRODUÇÃO}

Em dezembro de 2019 foi descoberto um novo Coronavírus (SARS-CoV-2) (1), o qual é responsável pela atual pandemia por COVID19 (2). O forte impacto multifacetado da pandemia imprimiu alterações profundas ao nível da saúde (3), da economia (4) e da sociologia (5), o qual se tem manifestado nas duas vagas reportadas até ao momento (6).

A infeção por COVID19 traduz-se por diversos sintomas (7), sendo os mais frequentes a tosse, a sensação de cansaço e a febre. A falta de ar, a dor no peito e a intolerância à atividade são considerados sintomas graves e indiciam a evolução para a forma mais severa da doença. São reportados outros sintomas como perda de olfato e paladar, conjuntivite, diarreia, dor de cabeça, erupções cutâneas ou dor osteoarticular. Sob o ponto de vista patológico, a pneumonia por COVD19 (8) é a principal entidade com relação direta com a infeção, embora os eventos tromboembólicos se afigurem como um dos eventos clínicos de risco na fase pós-aguda da doença (9).

Epidemiologicamente, a severidade da infeção por COVID19 tende a ter uma relação proporcional e direta com a idade, sendo essa relação de maior expressividade na população sénior (10). É também assumida uma maior suscetibilidade à doença nos indivíduos acometidos por doenças crónicas, principalmente do foro respiratório ou cardiovascular (11).

As medidas de combate ao COVID19 têm-se centrado em dois pólos: a) assistência precoce e efetiva aos infetados que apresentem forma grave de doença (12) e b) medidas de mitigação do contágio (13), das quais se destacam o isolamento social, o acesso condicionado a locais públicos e as medidas de etiqueta respiratória e de higienização das mãos. Porém, o isolamento social aliado ao acesso condicionado aos locais públicos tem diminuído a acessibilidade da população aos cuidados 
de saúde (14), o que se tem traduzido no agravamento das situações clínicas crónicas (15) e, consequentemente, no aumento das necessidades de cuidados de saúde.

Pelo descrito, o impacto da pandemia tem sido também muito sentido pelos profissionais de saúde, os quais relatam sobrecarga de trabalho (16) por aumento das necessidades de cuidados e por diminuição dos recursos humanos disponíveis, escassez de equipamentos de proteção individual (EPIs) (17), escassez de material clínico, exaustão física e mental (18), dificuldade nos processos de tomada de decisão clínica, entre outros.

É ainda relatada, como consequência da pandemia, a reorganização dos cuidados de saúde em função do enfoque na resposta direta aos casos COVID19 (19), o que tem diminuído a prestação de cuidados a outras patologias ou em outros contextos; são exemplo disso os cuidados de reabilitação (20), os quais têm sido preteridos em prol da manutenção da resposta adequada aos doentes com instabilidade clínica.

Torna-se, assim, pertinente conhecer o impacte da pandemia por COVID19 na prestação de cuidados de Enfermagem de reabilitação em Portugal, o qual não se encontra ainda aferido. De forma a contribuir para a construção desse conhecimento, e aproveitando uma iniciativa europeia de avaliação do impacto da pandemia COVID19 nos profissionais de saúde, definimos o seguinte objetivo: avaliar o impacte que a pandemia por COVID 19 teve sobre os cuidados de enfermagem prestados aos doentes, a satisfação profissional dos enfermeiros com os cuidados prestados, e identificar os desafios éticos enfrentados com maior frequência, bem como os recursos de treino e formação utilizados pelos enfermeiros.

\section{MÉTODO}

Para cumprir o objetivo proposto, realizámos um observacional, descritivo e transversal. Utilizámos uma amostra não probabilística de enfermeiros Portugueses associados da Associação Portuguesa de Enfermeiros de Reabilitação (APER). Utilizamos como critério de inclusão dos participantes, cumulativamente serem enfermeiros especialistas em Enfermagem de reabilitação, associados da APER, terem desempenhado funções em qualquer tipologia de serviço (ex.: internamento, ambulatório, cuidados primários) nos três meses anteriores à recolha de dados, e terem aceite participar no estudo. A recolha de dados realizou-se no início do terceiro trimestre de 2020.

A recolha de dados foi realizada através de um questionário online disponibilizado por email. 0 questionário foi desenvolvido por uma equipe de investigadores da Universidade de Torino em Itália, liderado pelo Professor Marco Clari. A validade de conteúdo foi assegurada pelos investigadores de todos os contextos envolvidos. 0 questionário era constituído por 6 secções. A primeira secção dirigia-se à caraterização sociodemográfica, a segunda à caraterização do impacte na organização do trabalho, a terceira à caraterização do impacte nos cuidados de enfermagem, a quarta à caraterização dos desafios éticos, a quinta à caraterização do impacte nos enfermeiros e no trabalho ao nível da instituição de cuidados e a sexta à caraterização do impacte nas atividade e recursos formativos. Em cada secção os itens foram avaliados através de uma escala de Likert de 5 pontos (1-pior opinião, 5-melhor opinião).

$\mathrm{Na}$ análise dos dados utilizamos a estatística descritiva com recurso software IBM SPSS Statistics (v.27).

\section{RESULTADOS}

A amostra deste estudo é constituída por 146 enfermeiros especialistas em Enfermagem de Reabilitação com uma idade média de 43,9 anos $(S D \pm 7,37 ; \quad M i n=28 ; \operatorname{Max}=61), 71,2 \% \quad(n=104)$ do sexo feminino. Dos participantes $35,6 \%(n=52)$ são mestres, $89,7 \% \quad(n=131)$ são enfermeiros especialistas das instituições, 6,8\% (n=10) são gestores e 3,4\% (=5) são enfermeiros de cuidados gerais nas instituições. Os enfermeiros têm em média 20,32 anos de experiência profissional (SD \pm 7,17; Min=6; $M a x=38)$.

Dos enfermeiros especialistas da amostra 96,6\% (n=141) têm um contrato por tempo indeterminado com a instituição prestadora de cuidados de saúde. Destes $90,1 \%(n=127)$ exercem funções em instituições do Serviço Nacional de Saúde. Dos enfermeiros especialistas da amostra 63,69\% (n=93) exercem funções em instituições hospitalares e os restantes na comunidade.

$\mathrm{Na}$ tabela 1 podemos observar a distribuição dos enfermeiros especialistas em Enfermagem de Reabilitação antes e durante a pandemia em relação ao exercício dos cuidados enfermagem.

Tabela 1 - Distribuição dos enfermeiros especialistas em Enfermagem de Reabilitação antes e durante a pandemia em relação ao exercício dos cuidados enfermagem.

\begin{tabular}{|l|c|c|}
\hline \multicolumn{1}{|c|}{$\begin{array}{c}\text { Cuidados de } \\
\text { Enfermagem }\end{array}$} & $\begin{array}{c}\text { Antes o início } \\
\text { da pandemia }\end{array}$ & $\begin{array}{c}\text { Após o início } \\
\text { da pandemia }\end{array}$ \\
\hline Gerais & $17,8 \%(n=26)$ & $48,6 \%(n=71)$ \\
\hline $\begin{array}{l}\text { Especializados em } \\
\text { tempo parcial }\end{array}$ & $42,5 \%(n=62)$ & $28,1 \%(n=41)$ \\
\hline $\begin{array}{l}\text { Especializados em } \\
\text { tempo integral }\end{array}$ & $39,7 \%(n=58)$ & $23,3 \%(n=34)$ \\
\hline
\end{tabular}

De realçar na tabela 1 o aumento em 2,7 vezes no número EEER que prestaram cuidados gerais no período do primeiro pico pandémico.

Relativamente aos cuidados de enfermagem nos três meses anteriores à recolha de dados (abril-junho) 31,5\% $(n=46)$, os participantes referiram terem prestado cuidados a pessoas com COVID 19. Nos serviços destes participantes em média estiveram internadas 14,81 pessoas com COVID por semana (SD $\pm 18,85$; Min=1; Max $=100$ ) e estes participantes prestaram cuidados de enfermagem em média 6,21 (SD $\pm 7,02 ; M i n=1 ; M a x=30)$ pessoas com COVID por turno. Nos contextos de cuidados com pessoas com COVID, dos participantes apenas $43,5 \%(n=20)$ prestaram cuidados especializados 
de enfermagem de reabilitação os restantes 56,5\% (26) prestaram cuidados gerais de enfermagem. Nesta amostra $21,9 \%(n=32)$ dos participantes referiram ter mudado de serviço durante a pandemia.

Durante este período $63,7 \%(n=93)$ dos participantes referiram que não existiram mudanças no número de doentes a que prestou cuidados por turno, 15,1\% ( $n=22)$ referiu que aumentou o número de doentes por turno a quem prestou cuidados e $21,2 \%(n=31)$ referiu que 0 número de doentes por turno diminuiu.

Antes do início da pandemia 50,7\% (n=74) dos participantes referiram ter experiência em cuidados intensivos, destes $29,7 \%$ (22) referiu ter muita experiência (Likert $=5)$. Dos participantes, 99,3\% $(n=145)$ referiram ter experiência com pessoas com doença respiratória, sendo deste $68,9 \% \quad(n=100)$ referiram uma perceção de experiência entre 4 e 5 na escala de Likert.

Dos participantes, $42,5 \%(n=62)$ referiram terem sido testados para a COVID19. No global dos participantes apenas $0,02 \%$ revelaram ter testado positivo $(n=3)$.

Em relação ao impacte na organização do trabalho os participantes revelaram uma perceção média de 2,78 $(S D \pm 0,70)$ sobre a existência de tempo das instituições para se prepararem para a resposta à pandemia, e uma perceção média de $2,6(S D \pm 0,67)$ relativamente ao tempo para a formação dos enfermeiros.

A pandemia esteve na origem de mudanças no exercício profissional e na vida pessoal dos enfermeiros especialistas em enfermagem de reabilitação. Em relação ao tempo para a preparação e organização da vida pessoal os participantes manifestaram um valor médio de 2,53 (SD $\pm 0,66)$. Dos participantes $17,8 \%$ $(n=26)$ referiram que tiveram de mudar de residência durante a pandemia.

Na tabela 2 apresentamos os valores médios do impacte das mudanças nas dimensões dos cuidados de enfermagem e da sua articulação com a vida pessoal. Verificando-se que é na complexidade dos doentes, no relacionamento com os colegas de trabalhos, no balanço entre a vida profissional e pessoal e nas atividades burocráticas que mais participantes referiram terem existindo mudanças. Das mudanças mais frequentes a que maior impacte teve foi a relativa ao balanço entre a vida profissional e pessoal.

Relativamente à satisfação com a qualidade dos cuidados prestados verificamos que a média antes do início da pandemia situava-se em 3,95 (SD $\pm 0,75)$ e durante a pandemia desceu para $2,9 \quad(S D \pm 1,11)$ [avaliado através de uma escala de Likert de 5 pontos (1-pior opinião, 5-melhor opinião)].

Relativamente aos desafios éticos com os quais os participantes se confrontaram, 73,3\% $(n=107)$ referem que em algum momento tiveram de seguir orientações institucionais em desacordo com os seus princípios éticos e deontológicos. Dos participantes $69,9 \%(n=102)$ refere que em algum momento da pandemia teve de priorizar a que pessoas doentes prestar cuidados.
Tabela 2 - Impacte das mudanças em diferentes dimensões dos cuidados de enfermagem.

\begin{tabular}{|c|c|c|c|}
\hline Itens & $\mathbf{N}$ & $\mathrm{X}$ & SD \\
\hline Rácio de assistentes operacionais & 74 & 3,04 & 0,73 \\
\hline Organização do espaço de trabalho & 77 & 3,29 & 0,70 \\
\hline Complexidade dos doentes & 90 & 3,11 & 0,71 \\
\hline Enfermeiros por Turnos & 69 & 3,16 & 0,7 \\
\hline $\begin{array}{l}\text { Utilização de equipamentos de } \\
\text { proteção individual }\end{array}$ & 51 & 3,33 & 0,68 \\
\hline $\begin{array}{l}\text { Relacionamento com familiares dos } \\
\text { doentes }\end{array}$ & 58 & 3,22 & 0,77 \\
\hline $\begin{array}{l}\text { Relacionamento com colegas de } \\
\text { trabalho }\end{array}$ & 87 & 3,16 & 0,73 \\
\hline $\begin{array}{l}\text { Balanço entre a vida profissional e } \\
\text { pessoal }\end{array}$ & 84 & 3,42 & 0,68 \\
\hline Treino e integração de enfermeiros & 65 & 3,15 & 0,73 \\
\hline $\begin{array}{l}\text { Supervisão de estudantes de } \\
\text { enfermagem }\end{array}$ & 26 & 3,08 & 0,8 \\
\hline Atividades burocráticas & 80 & 3,13 & 0,79 \\
\hline
\end{tabular}

$\mathrm{Na}$ tabela 3 apresentamos os valores médios da utilização de diferentes recursos de aprendizagem dos participantes e o seu impacte na gestão e organização dos cuidados.

Tabela 3 - Recursos utilizados e o seu impacte na aprendizagem durante a pandemia

\begin{tabular}{|c|c|c|c|c|}
\hline \multirow{2}{*}{$\begin{array}{l}\text { Recursos utilizados na } \\
\text { aprendizagem }\end{array}$} & \multicolumn{2}{|c|}{ Utilização } & \multicolumn{2}{|c|}{ Impacte } \\
\hline & $\mathrm{X}$ & SD & $X$ & SD \\
\hline Colegas peritos & 3,22 & 0,67 & 3,22 & 0,69 \\
\hline Outros peritos da equipe de saúde & 3,09 & 0,72 & 3,22 & 0,67 \\
\hline Webinars & 3,09 & 0,72 & 3,17 & 0,61 \\
\hline Websites institucionais & 3,29 & 0,66 & 3,07 & 0,69 \\
\hline Plataformas de e-learning & 3,15 & 0,65 & 3,20 & 0,66 \\
\hline Jornais e revistas científicas online & 2,77 & 0,68 & 3,19 & 0,70 \\
\hline $\begin{array}{l}\text { Redes sociais (ex.: } \quad \text { Facebook, } \\
\text { Twitter, WhatsApp ...) }\end{array}$ & 3,22 & 0,67 & 2,76 & 0,68 \\
\hline
\end{tabular}

\section{DISCUSSÃO}

Este estudo revelou que os enfermeiros Portugueses especialistas em Enfermagem de Reabilitação (EEER) durante o surto inicial e o primeiro pico pandémico tiveram de se adaptar aos novos desafios organizacionais, pessoais, éticos e formativos. Durante a pandemia os desafios enfrentados conduziram à necessidade de muitos EEER passarem a prestar apenas cuidados gerais para colmatar défices de enfermeiros verificados nos serviços. De realçar, contudo, que $51,4 \%$ dos EEER da amostra mantiveram-se a prestar cuidados especializados, aspeto que atesta a sua relevância para assegurar a qualidade e segurança dos cuidados gerais 
e especializados. Este facto é corroborado pela elevada experiência em ambiente de cuidados a pessoas em estado crítico e a pessoas com patologia respiratória.

A transição de EEER para os cuidados gerais poderá justificar-se numa situação de emergência pandémica; contudo, devemos realçar que para que os EEER possam exercer cuidados gerais, as necessidades de cuidados especializados têm necessariamente de deixar de ser assegurados. Este facto coloca em causa a qualidade, a segurança e os resultados dos cuidados de saúde. Assim, este estudo alerta-nos para o facto da solução encontrada pelos gestores, num primeiro pico pandémico, de assegurar cuidados gerais através da alocação de EEER concorrer para a redução da resposta em termos de cuidados especializados em enfermagem de reabilitação e, consequentemente, para uma detioração da qualidade global dos cuidados de saúde disponibilizados à população portuguesa.

Este estudo revela ainda que, neste período, a complexidade da condição das pessoas doentes aumentou, bem como os aspetos burocráticos que suportam a gestão dos cuidados. De realçar que o relacionamento com os colegas de trabalho foi um dos aspetos com maior impacte neste período. Este dado não é alheio à utilização de equipamentos de proteção individual (EPI), à separação de circuitos e à necessidade dos EEER coordenarem e articularem os cuidados de enfermagem especializados com equipe de saúde dos contextos de cuidados.

A necessidade de reorganizar os cuidados de saúde para prestar cuidados às pessoas com COVID conduziu a uma redução da satisfação dos EEER com os cuidados de enfermagem prestados. Neste estudo deve salientar-se que $84,9 \%$ dos participantes referem ter mantido ou até reduzido o número de doentes por turno, o que pode traduzir uma menor prestação de cuidados. Estes dados podem ajudar a compreender a redução da satisfação com os cuidados, quer pela impossibilidade de prestar cuidados especializados, quer pelas alterações nas dinâmicas relacionais e técnicas impostas pelas medidas de prevenção da contaminação.

Este estudo identifica uma elevada percentagem EEER que em algum momento, deste período, tiveram de seguir orientações institucionais em desacordo com os seus princípios éticos e deontológicos. Este estudo não permite explorar este dado, contudo não deixamos de realçar a nossa preocupação. Por este motivo torna-se de extrema relevância estudar este facto para poder implementar medidas corretivas com a maior urgência.

Este estudo permite-nos perceber que os EEER pelos desafios que enfrentaram procuraram diferentes tipos de recursos de aprendizagem que tiveram impacte na formação e consequentemente na qualidade e segurança dos cuidados de enfermagem. Estes dados ajudam-nos, também, a perceber o dinamismo formativo e o interesse que os EEER apresentam para utilizar as tecnologias da informação e comunicação como recurso formativo.

Como principais limitações deste estudo identificamos o reduzido número de participantes e a inexistência de dados comparativos a nível nacional ou internacional que nos permitam uma discussão mais aprofundada.

\section{CONCLUSÃO}

Durante o primeiro pico pandémico por COVID 19 os EEER enfrentaram desafios para poderem garantir a continuidade, qualidade e segurança dos cuidados de enfermagem especializados. Neste período muitos dos EEER tiveram de assegurar apenas cuidados gerais. Os principais desafios enfrentados pelos EEER foram no domínio da organização e gestão dos cuidados devido à maior complexidade dos doentes, à maior carga burocrática, às mudanças no relacionamento com os colegas de trabalhos e à necessidade de balanço entre a vida profissional e pessoal. É de realçar a diminuição da satisfação com a qualidade dos cuidados prestados, bem como a elevada percentagem de EEER que vivenciou desafios éticos e deontológicos. Relativamente aos recursos de aprendizagem e o seu impacte na formação, os EEER revelaram dinamismo e apetência para a utilização das tecnologias da informação e comunicação.

\section{REFERÊNCIAS BIBLIOGRÁFICAS}

1. Di Gennaro F PDMCAMRVVNea. Coronavirus Diseases (COVID-19) Current Status and Future Perspectives: A Narrative Review. International journal of environmental research and public health. $2020 \mathrm{Apr}$.

2. WHO. WHO. [Online].; 2020. Available from: https://www.who.int/emergencies/diseases/novel-coronavirus2019?gclid=CjOKCQiA2uH-BRCCARIsAEeef3nQCzCLFKtHyrqCcxU08PxtUMONIQTJ-

_M840cJju5060YkkYKK64aAhUuEALw_wcB.

3. WHO. World health statistics 2020: monitoring health for the SDGs, sustainable development goals: World Health Organization; 2020.

4. World Bank Group. Pandemic, Recession: The Global Economy in Crisis. In Global Economic Prospects. Washington: International Bank for Reconstruction and Development / The World Bank; 2020.

5. Khurshid A AAMAAAR. Living with Coronavirus (COVID-19): a brief report. European review for medical and pharmacological sciences. 2020 Oct: p. 10902-12.

6. Cacciapaglia G,CC\&SF. Second wave COVID-19 pandemics in Europe: a temporal playbook. Sci Rep. 2020 Sep.

7. WHO..; 2020 [cited 2020 Nov 15. Available from: https://www.who.int/health-topics/coronavirus\#tab=tab_3.

8. Zhang Y LYGHWL. Quantitative lung lesion features and temporal changes on chest CT in patients with common and severe SARSCoV-2 pneumonia. PloS one. 2020 Jul.

9. Allegra A IVAAMC. Coagulopathy and thromboembolic events in patients with SARS-CoV-2 infection: pathogenesis and management strategies. Annals of Hematology. 2020 Sep.

10. WHO. COVID-19 Weekly Epidemiological Update - Nov 1st 2020. World Health Organization; 2020.

11. CDC. People with Certain Medical Conditions. [Online].; 2020 [cited 2020 Nov 15. Available from: https://www.cdc.gov/coronavirus/2019-ncov/need-extraprecautions/people-with-medical-conditions.html.

12. NIH. Coronavirus Disease 2019 (COVID-19): Treatment Guidelines. [Online].; 2020 [cited 2020 Nov 15. Available from: https://www.covid19treatmentguidelines.nih.gov/.

13. Haj Bloukh S EZSAPHA. Look Behind the Scenes at COVID-19: National Strategies of Infection Control and Their Impact on Mortality. International journal of environmental research and public health. 2020 Aug.

14. Bartolomé Benito E SVMMHIMSJ. Proposals by the Spanish Society of Quality in Healthcare (SECA) for the recovery of the National Health Service after the COVID-19 pandemic. Journal of healthcare quality research. 2020 Oct.

15. Elran-Barak R MM. One Month into the Reinforcement of Social Distancing due to the COVID-19 Outbreak: Subjective Health, 
Health Behaviors, and Loneliness among People with Chronic Medical Conditions. International journal of environmental research and public health. 2020 Jul.

16. Morgantini LA NUWHFSAÖFJea. Factors contributing to healthcare professional burnout during the COVID-19 pandemic: A rapid turnaround global survey. PloS one. 2020 Sep.

17. Mantelakis A SHLCCAJA. Availability of Personal Protective Equipment in NHS Hospitals During COVID-19: A National Survey. Annals of work exposures and health. 2020 Sep.

18. Bozdağ F EN. Psychological Resilience of Healthcare Professionals During COVID-19 Pandemic. Psychological reports. 2020 Oct.
19. WHO. Maintaining essential health services: operational guidance for the COVID-19 context: World Health Organization; 2020.

20. Genovese TJ CAPJ. Physical Medicine and Rehabilitation in Rhode Island during the COVID-19 Pandemic. Rhode Island medical journal. 2020 Nov. 